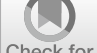

Check for

updates

Cite as

Nano-Micro Lett.

(2020) $12: 160$

Received: 9 May 2020

Accepted: 22 June 2020

Published online: 8 August 2020

(C) The Author(s) 2020

\section{Parallel Nanoimprint Forming of One-Dimensional Chiral Semiconductor for Strain-Engineered Optical Properties}

\author{
Yixiu Wang ${ }^{1,2}$, Shengyu Jin ${ }^{1,2}$, Qingxiao Wang ${ }^{3}$, Min Wu ${ }^{1,2}$, Shukai Yao ${ }^{4}$, Peilin Liao ${ }^{4}$, \\ Moon J. Kim ${ }^{3}$, Gary J. Cheng ${ }^{1,2}$, Wenzhuo $\mathrm{Wu}^{1,2,5,6} \bowtie$ \\ Yixiu Wang and Shengyu Jin contributed equally to this work. \\ $\triangle$ Gary J. Cheng, gjcheng@purdue.edu; Wenzhuo Wu, wenzhuowu@purdue.edu \\ 1 School of Industrial Engineering, Purdue University, West Lafayette, IN 47907, USA \\ 2 Flex Laboratory, Purdue University, West Lafayette, IN 47907, USA \\ 3 Department of Materials Science and Engineering, University of Texas at Dallas, Richardson, TX 75080, \\ USA \\ 4 School of Materials Engineering, Purdue University, West Lafayette, IN 47907, USA \\ 5 Birck Nanotechnology Center, Purdue University, West Lafayette, IN 47907, USA \\ 6 Regenstrief Center for Healthcare Engineering, Purdue University, West Lafayette, IN 47907, USA
}

\title{
HIGHLIGHTS
}

- Exquisite strain engineering in 1D chiral semiconductor.

- Facile nanoimprinting induced tensile strain in Te nanowire.

- Intriguing and tunable optical properties of 1D Te nanowire by strain engineering.

\begin{abstract}
The low-dimensional, highly anisotropic geometries, and superior mechanical properties of one-dimensional (1D) nanomaterials allow the exquisite strain engineering with a broad tunability inaccessible to bulk or thin-film materials. Such capability enables unprecedented possibilities for probing intriguing physics and materials science in the 1D limit. Among the techniques for introducing controlled strains in 1D materials, nanoimprinting with embossed substrates attracts increased attention due to its capability to parallelly form nanomaterials into wrinkled structures with controlled periodicities, amplitudes, orientations at large scale with nanoscale resolutions. Here, we systematically investigated the strain-engineered anisotropic optical properties in Te nanowires through introducing a controlled strain field using a resist-free thermally assisted nanoimprinting process. The magnitude of induced strains can be tuned by adjusting the imprinting pressure, the nanowire diameter, and the patterns on the substrates. The observed Raman spectra from the chiral-chain lattice of 1D Te reveal the

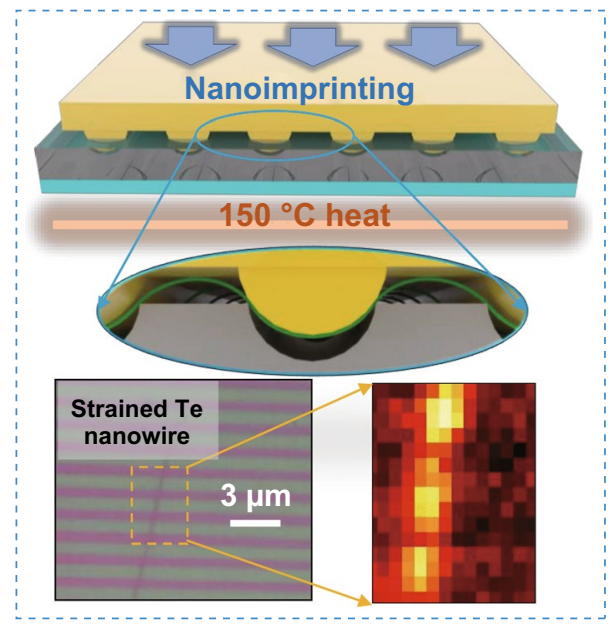
strong lattice vibration response under the strain. Our results suggest the potential of 1D

Te as a promising candidate for flexible electronics, deformable optoelectronics, and wearable sensors. The experimental platform can also enable the exquisite mechanical control in other nanomaterials using substrate-induced, on-demand, and controlled strains.
\end{abstract}

KEYWORDS Chiral semiconductor; Nanowires; Nanoimprinting; Strain engineering; Optical property 


\section{Introduction}

The low-dimensional, highly anisotropic geometries, and superior mechanical properties of one-dimensional (1D) nanomaterials [1-3] allow the introduction of enormous elastic strains [3-7] (e.g., > 1\%) and even designer strain fields [8-11] without fracture. These materials are amenable to exquisite mechanical engineering $[9,12,13]$ with a wide range of tunability inaccessible to bulk or thin-film materials. Such engineering capability offers unprecedented possibilities for probing intriguing physics and materials science in the 1D limit [14-17]. The strong coupling of mechanical strain to various internal degrees of freedom involving charges, photons, spins, etc., also enables the design and implementation of device technologies with novel functionalities, such as stretchable electronics/optoelectronics [18-21], quantum straintronics [22-25], electromechanical sensors [26-28], strain-engineered piezotronics [29-32], and mechanically enhanced catalysis [33-35]. Strain engineering in $1 \mathrm{D}$ semiconductors such as silicon $[6,7,36]$ and $\mathrm{ZnO}[29$, 31,37 nanowires has been explored theoretically [38-44] and experimentally $[7,36,45,46]$ as an effective approach to rationally engineer the crystal structure, semiconductor properties, and device functions of the related materials [47-54].

Several techniques have been demonstrated to be feasible for introducing controlled strains in 1D materials through, e.g., bending of the flexible substrates [55], elongating of the elastic substrates [56], thermally induced expansion of the substrates [57, 58], and embossing/imprinting the active materials to patterned substrates [59-62]. Among these strategies, imprinting the 1D nanowires into designed patterns attracts increased attention due to its capability to parallelly form the different segments of the materials into wrinkled structures with controlled periodicities, amplitudes, and orientations, closely following the predefined patterns on the host substrates. Compared to other nanopatterning schemes based on lithography techniques, such as photolithography [63], electron beam lithography (EBL) [64], focused ion beam lithography (FIBL) [65], nanoimprinting exhibits unique advantages for resist-free, high-resolution, low-cost, rapid, and high-throughput patterning [66-71]. Moreover, nanoimprinting can also introduce elastic strains in designed patterns with nanoscale resolution [66, 72, 73]. Nevertheless, much less was known for the application of nanoimprinting on the parallel forming of semiconductor nanowires.
As a Group VI element, bulk tellurium (Te) is a $p$-type semiconductor with a narrow bandgap of $0.35 \mathrm{eV}[74,75]$. Te exhibits interesting properties such as semiconducting $[76,77]$, thermoelectric [78], piezoelectric [79, 80] for application in electronics, energy devices, and sensors [81, 82]. Te's intriguing trigonal crystal lattice $[76,77,82,83]$ consists of anisotropic 1D chiral chains. Each Te atom is covalently bonded with its two nearest neighbors on the same chain, and the interchain interaction is weaker than the covalent bond [74]. A systematic study on strain engineering the 1D Te's anisotropic properties will be helpful for providing fundamental insights of the coupling between mechanical strains and various internal degrees of freedom in Te nanomaterials $[76,77,80]$ and other materials sharing similar 1D chain structures [84-86], as well as enabling the design and development of novel smart devices capable of actively interacting with the working environment $[31,87,88]$.

Here, we systematically investigated the strain-engineered anisotropic optical properties in 1D Te nanowires, through designing and introducing a controlled strain field in solution-grown ultralong Te nanowires, using a resist-free thermally assisted nanoimprinting method. The magnitude of induced strains can be tuned by adjusting the imprinting pressure, the nanowire diameter, and the patterns on the substrates. The observed Raman spectra from the chiralchain lattice of $1 \mathrm{D} \mathrm{Te}$, dependent on the magnitude of the introduced strain, reveal the strong lattice vibration response under the corresponding strain conditions. Our results suggest the potential of 1D Te as a promising candidate for enabling flexible electronics $[89,90]$, deformable optoelectronics [91, 92], and wearable sensors [26, 28, 31, 93]. The experimental platform can also enable the exquisite mechanical control in 1D Te and other 1D nanomaterials with substrate-induced, on-demand, and controlled strains.

\section{Results and Discussion}

Figure 1a shows the morphology of the solution synthesized ultralong Te nanowires (Methods) with lengths over hundreds of micrometers. The high-resolution transmission electron microscope (HRTEM) (Fig. 1b) characterization for the side edge of a Te nanowire reveals the atomically resolved lattice of Te nanowire. In Fig. 1b, the measured lattice spacings of 5.9 and $3.9 \AA$ correspond to the (0001) and (1010) planes for trigonal tellurium, respectively, which matches 

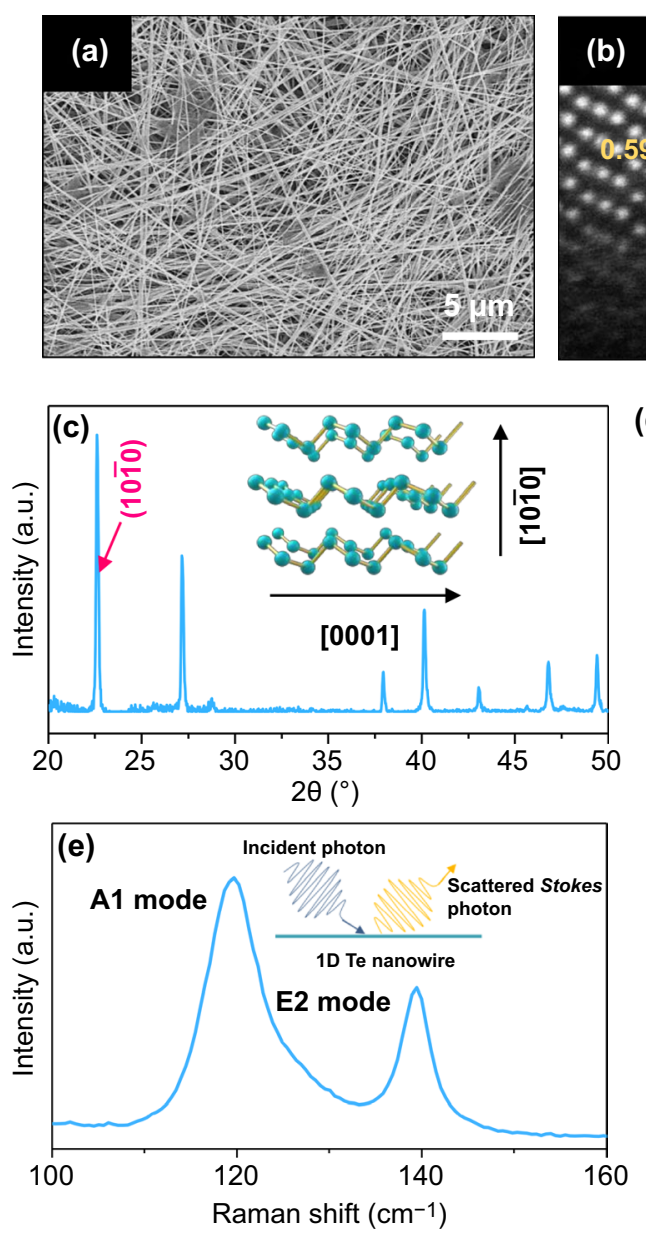

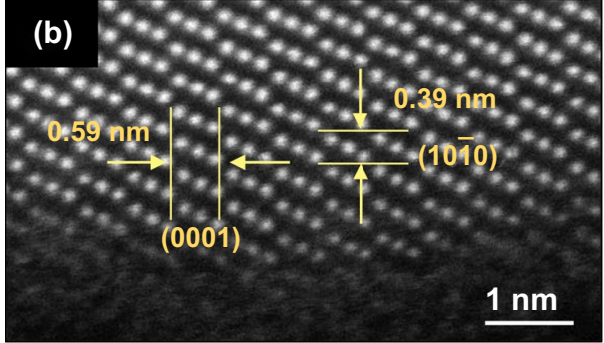

(d)
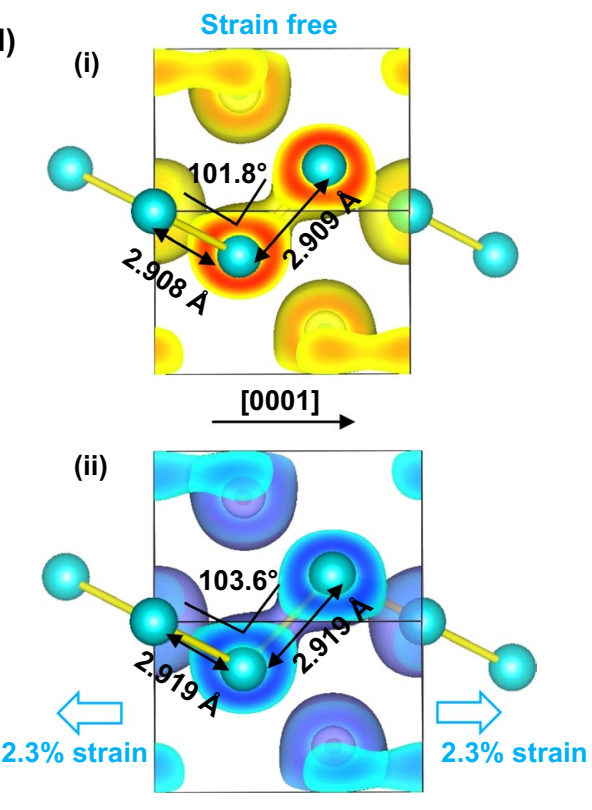

Fig. 1 Characterization of 1D Te nanowire. a Scanning electron microscopy (SEM) image of the as-synthesized 1D Te nanowire. b High-resolution transmission electron microscope image of the 1D Te nanowire at the edge. $\mathbf{c}$ XRD results of the 1D Te nanowire. $\mathbf{d}$ Atomic structures of the Te crystals without and with tensile strain. The isosurface value for electron density is $0.03 \mathrm{e} / \mathrm{Bohr}^{3}$, e Raman spectra of the 1D Te nanowire

well with the previous reports [94, 95]. The HRTEM results also suggest the crystal orientation for the nanowires' length directions is along [0001]. X-ray powder diffraction (XRD) results of the powdered Te nanowires sample (Fig. 1c) further verify that the product consists of crystalline elemental tellurium. All the diffraction peaks can be indexed as the trigonal tellurium phase (JCPDS No. 36-1452) [94, 96]. The high relative intensity of (1010) diffraction peaks indicates the top surface of 1D Te nanowires are dominated by the (10ī0) planes (see the atomic structure in Fig. 1c insert). It should be noted that the helical chains of Te atoms (hence a threefold screw symmetry) are packed along the longitudinal direction of Te nanowires ([0001] direction), and the radial stacking in the Te nanowire is along the [1010] direction. We performed density functional theory (DFT) calculations to explore the difference in the optimized structures without and with tensile strains (see Methods). As is shown in Fig. 1d(i), the bond distances between Te and its nearest Te atoms along the [0001] chain direction are $2.909 \AA$, and the angle between the two adjacent bonds is $101.9^{\circ}$. After $2.3 \%$ tensile strain was applied along the [0001] direction, the bond distances and angle change to $2.919 \AA$, $2.918 \AA$, and $103.5^{\circ}$, respectively. In Fig. 1d(ii), we also marked the electron density with $0 \%$ (yellow) and $2.3 \%$ (blue) tensile strain. Such structural change could lead to a significant change in the lattice vibration. It is well understood that Raman spectroscopy can sensitively reveal the surface vibration of the sample (Fig. 1e insert). Figure 1e shows the Raman spectra of a Te nanowire sample with a diameter larger than $40 \mathrm{~nm}$. The Raman signal was excited by a $633-\mathrm{nm}$ laser along the 
[1010] direction of Te nanowires. Two Raman modes locating at $121 \mathrm{~cm}^{-1}$ and $143 \mathrm{~cm}^{-1}$ can be identified, which agree well with the previous results for bulk tellurium [76, 97]. Further detailed exploration for strain-engineered Raman spectra of 1D Te will be discussed in later sections.

A schematic configuration illustrating the process scheme for nanoimprinting induced parallel forming of Te nanowires is shown in Figs. 2a-c. To minimize the undesired entanglement and agglomeration (Fig. 1a) of the as-synthesized ultralong, thin Te nanowires for ensuring a controlled straining process, we first performed a Langmuir-Blodgett (LB) process $[98,99]$ to transfer and assemble the Te nanowires from the synthesis solution into a monolayer assembly [100] on a soft polyester terephthalate (PET) substrate. Figure 2d shows a TEM image of the LB-assembled Te nanowires on a copper grid. Furthermore, such an LB assembly process facilitates the orientation alignment of the constituent nanowires in a large-scale assembly [101] (Fig. 2a, d). The statistic histogram in Fig. 2e shows that the as-synthesized
Te nanowires possess diameters from 35 to $70 \mathrm{~nm}$. Through tuning the pressure of imprinting (method), the aligned Te nanowires can be stamped onto mold substrates with periodic gratings (blank CD or DVD disk), which induces a periodic strain field in the Te nanowire (Fig. 2c). The magnitude of the strain can be engineered by adjusting the pressure power, the diameter of the nanowire, and using CD or DVD substrates. Figure $2 \mathrm{~b}$ illustrates the nanoimprint-induced straining process. The resist-free thermal nanoimprinting process is a pattern emboss method. When the mold indents the PET at relatively low pressure (e.g., $1 \mathrm{MPa}$ ) with heat, the plastic flow of the PET substrate allows the mold to move inwards the PET, which induces a controllable local strain in the segment of Te nanowire sandwiched in between. The selection of suitable molding substrates with patterned surfaces is crucial for this method [59]. The commercial compact disk (CD) and digital versatile disk (DVD) encode their information by a spiral track molded onto the top of a polycarbonate layer [102]. The spiral tracks on blank CDs (a)

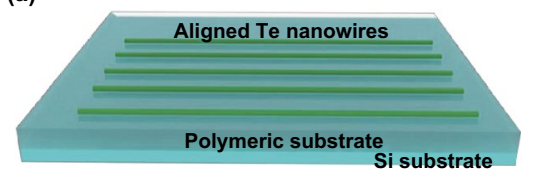

(b)

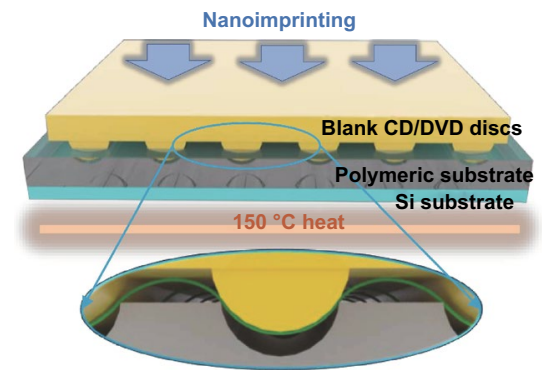

(c)

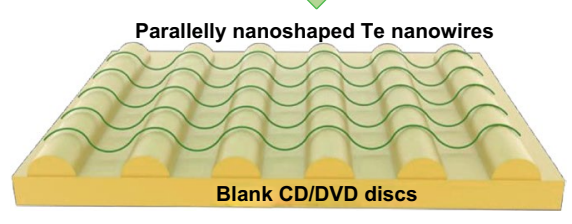

(d)

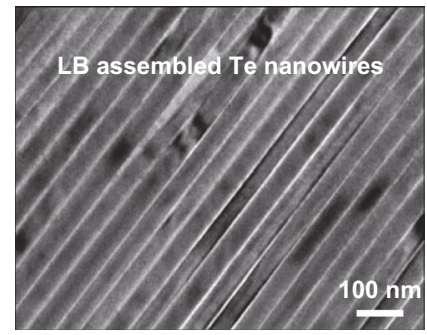

(f)

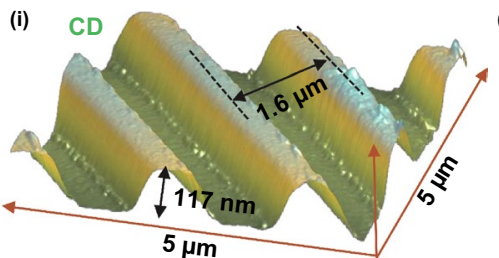

(g) $1 \mathrm{MPa}$ Pressure

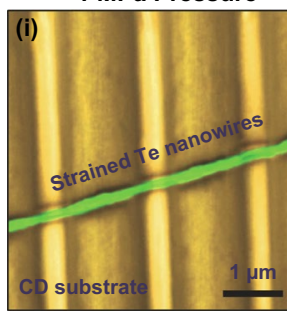

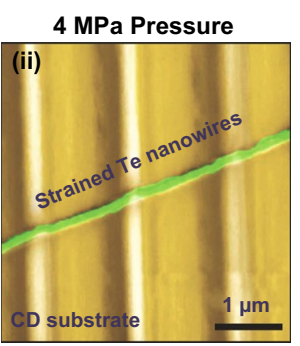

(e)

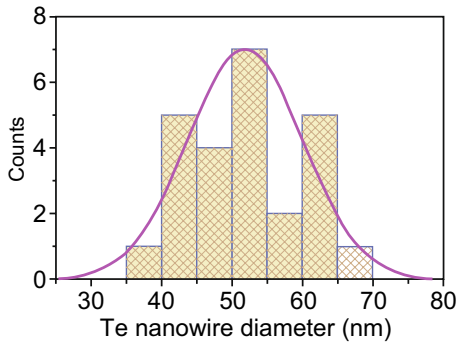

(ii)

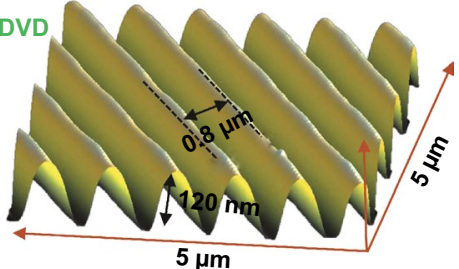

(h)

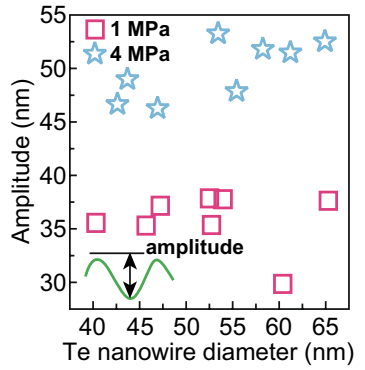

Fig. 2 Fabrication and topography characterization of the parallelly deformed 1D Te. a-c Schematic procedure of fabricating the wavy geometry of 1D Te nanowire on optical disks. $\mathbf{d}$ Transmission electron microscope image of the assembled 1D Te nanowire on the copper grid. e The thickness distribution of the as-synthesized 1D Te nanowire. f Atomic force microscopes (AFM) image of the optical disks. $\mathbf{g}$ Scanning electron microscope images of the deformed 1D Te nanowire by 1 and $4 \mathrm{MPa}$ nanoimprinting pressure. $\mathbf{h}$ The relationship between the deformed amplitude and Te nanowire diameter 
and DVDs have depths around hundreds of nanometers, and the distance between each neighboring track varies from $850 \mathrm{~nm}$ to $2 \mathrm{um}$ [102]. The surface patterns with precise periodicities and low cost of blank CD and DVD make them ideal substrates for the nanoimprinting process. The nanoimprint strained Te nanowires, supported on the CD/DVD substrate, is expected to exhibit a sinusoidal shape where the wavelength of the strain field in Te nanowire equals to the distance of the neighboring indentations in the CD or DVD substrates (Fig. 2c), and the amplitude of the strain field is primarily determined by the pressure of the nanoimprinting process [67, 69, 71].

Atomic force microscope (AFM) was used to characterize the topography (in terms of the periodicity and the depth of the mold) of the starting blank CD [Fig. 2f(i)] and DVD substrates [Fig. 2f(ii)]. The results show that the grating depth and periodicity are $117 \mathrm{~nm}$ and $1.6 \mu \mathrm{m}$ for the CD and $120 \mathrm{~nm}$ and $0.8 \mu \mathrm{m}$ for the DVD molds, respectively. The scanning electron microscopy (SEM) images in Fig. $2 \mathrm{~g}$ illustrate a Te nanowire (diameter $\sim 50 \mathrm{~nm}$ ), which is parallelly formed on a CD substrate with different nanoimprinting pressures. We optimized the process temperature at $150{ }^{\circ} \mathrm{C}$ and kept this value for all nanoimprinting experiments in this work. We used two nanoimprinting pressures (1 and $4 \mathrm{MPa}$ ) in our experiment. As is shown in the false-color SEM images in Fig. 2g, the bright gold areas are the top surfaces of the indentations on the CD mold, and the dark gold areas are the valley areas between these indentations. We did not observe cracking or fracture in the Te nanowire for either the low-pressure (1 MPa) or the high-pressure (4 MPa) nanoimprinting process, which indicates the superior mechanical property of crystalline Te nanowire [79, 84]. Figure $2 \mathrm{~h}$ summarizes the measured deformation amplitudes of the sinusoidal shape 1D Te nanowire by AFM with different nanoimprinting pressures. We examined 8 nanowires with different diameters for each pressure. Under 1-MPa pressure, the amplitude ranges from 30 to $38 \mathrm{~nm}$, while the amplitude reaches to 45-55 $\mathrm{nm}$ with the 4-MPa pressure imprinting.

We also characterized the topological configuration of strained Te nanowires (diameter $\sim 45 \mathrm{~nm}$ ) on a CD mold (Fig. 3). The AFM line scans performed in the regions for the Te nanowire and the CD substrate (Fig. 3a) show the periodic sinusoidal shapes for both the strained Te nanowire and the CD substrate [Fig. 3b(i), (ii)]. The amplitude and wavelength for the sinusoidal shape Te nanowire (line 1) were determined to be $50 \mathrm{~nm}$ and $1.6 \mu \mathrm{m}$ under $4 \mathrm{MPa}$.
The amplitude and wavelength for the CD patterns (line 2) were determined to be $120 \mathrm{~nm}$ and $1.6 \mu \mathrm{m}$, respectively. The bending mechanics in the deformed Te nanowire can be estimated using the schematics shown in Fig. 3c, where a nanowire with a diameter of $h$ is bent to a sinusoidal curvature. The nanowire segment on top of the CD indentation is tensilely strained [103, 104]. In the regime where $h$ is small compared to the radius of the curvature $r(h=40-70 \mathrm{~nm}$ and $r=1-2.5 \mu \mathrm{m}$ ), the peak strain induced in the nanowire can be estimated by the equation:

$\varepsilon_{1 \mathrm{D} \mathrm{Te}}^{\text {peak }}=\frac{h}{2 r}$

Here, $r$ is the radius of curvature at the peak of the wavy, which is generally expressed by

$r=-\left.\frac{1}{y^{\prime \prime}}\right|_{x= \pm\left[\frac{(2 n-1) \pi}{2 k}\right]}$

where $n$ is an integer and $y^{\prime \prime}$ is the second derivative of $y$ with respect to $x$. The shape of the wavy 1D Te can be expressed by a sine function,

$y=(A / 2) \cdot \sin (k x)(k=2 \pi / \lambda)$

where $\mathrm{A}$ and $\lambda$ are amplitude and wavelength of the sinusoidal Te nanowire (Fig. 3c). As a result, the peak strain induced in the Te nanowire can be expressed as

$\varepsilon_{1 \mathrm{D} \mathrm{Te}}^{\mathrm{peak}}=\frac{\pi^{2} A h}{\lambda^{2}}$

where the amplitude $A$, diameter $h$, and wavelength $\lambda$ can all be determined from the AFM results (Fig. 3b). Figure $3 \mathrm{~d}$, e summarizes the derived local peak strains for the deformed Te nanowires on CD and DVD substrates with different imprinting pressures. In Fig. 3d, we examined the local strains of 16 different Te nanowires with diameters ranging from 35 to $65 \mathrm{~nm}$ on CD and DVD molds, when we maintained a $4 \mathrm{MPa}$ pressure for the nanoimprinting. The green circles represent the $8 \mathrm{Te}$ nanowires on the CD substrates, and the blue squares represent the $8 \mathrm{Te}$ nanowires on the DVD substrates. The local peak strains in Te nanowires increase with the increased nanowire diameters. For instance, the local peak strain is $0.8 \%$ for Te nanowire with a $36.6 \mathrm{~nm}$ diameter and increases to $1.9 \%$ for Te nanowire with a $64.8 \mathrm{~nm}$ diameter. Moreover, our results revealed that the use of DVD substrates could induce more significant local strains since the wavelength $\lambda$ of the deformed Te nanowires on DVD substrates is smaller than on CD substrates. Figure $3 e$ summarizes the results when two imprinting 

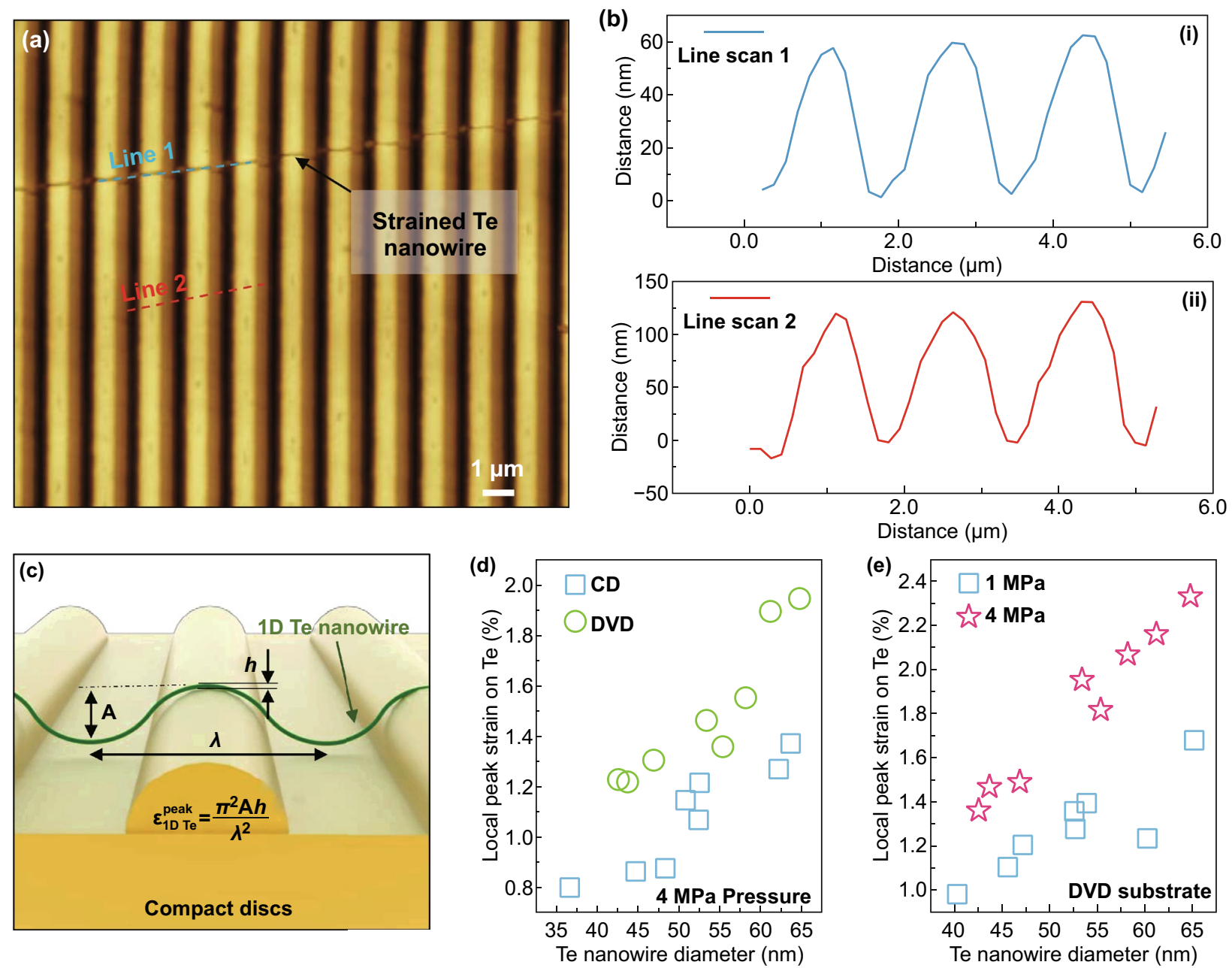

Fig. 3 Analysis of the deformed 1D Te on the optical disks. a Atomic force microscopes (AFM) image of the strained 1D Te nanowire on compact disks. b Two line-scan results from a. $\mathbf{c}$ The strain analysis of the deformed 1D Te nanowire on the compact disks. The relationship between the local peak strain and Te nanowire diameter $\mathbf{d}$ on different disks and $\mathbf{e}$ different nanoimprinting pressure

processes (1-MPa and 4-MPa) were applied to Te nanowires (diameters ranging from 40 to $65 \mathrm{~nm}$ ) on DVD substrates. The local peak strains increase with increased nanowire diameters. Also, a higher imprinting pressure (i.e., $4 \mathrm{MPa}$ ) will result in more significant local strains in the nanowires. For example, the local peak strain in a Te nanowire (diameter $\sim 65 \mathrm{~nm}$ ) is $1.7 \%$ when $1 \mathrm{MPa}$ pressure process was applied, and reaches to $2.3 \%$ when $4 \mathrm{MPa}$ pressure was applied. Consequently, we could rationally tune the local strains in the formed Te nanowires through manipulating the nanowire diameter, mold type (CD or DVD), and the imprinting pressure.

These parallelly nanoshaped tellurium nanowires with controlled local strains provide an ideal system to explore the strain-engineered optical property at the nanoscale. To this end, we characterized the strained 1D Te nanowires with Raman spectroscopy at room temperature. Figure $4 a$ illustrates the two vibration modes in the chiral-chain structure of Te. The $A^{1}$ mode represents the breathing vibration in the (1010) plane, and $E^{2}$ mode is the asymmetric stretching along [0001] direction in tellurium [76, 97] (Fig. 4a). We first performed Raman spectra mapping for the area enclosed by the orange dashed lines in the optical image in Fig. 4b. We found a periodic distribution of the Raman peak intensity of $A^{1}$ mode spanning across the entire nanowire, which is consistent with the topography variation in the strained nanowire (Fig. 4b). This result not only confirmed the periodic deformations in the strained nanowire but also helped us identify the regions with the local peak strains with nanoscale resolution. Subsequently, we examined the strain-induced shift in the Raman peaks from the positions where the local segments of the nanowire experienced the 


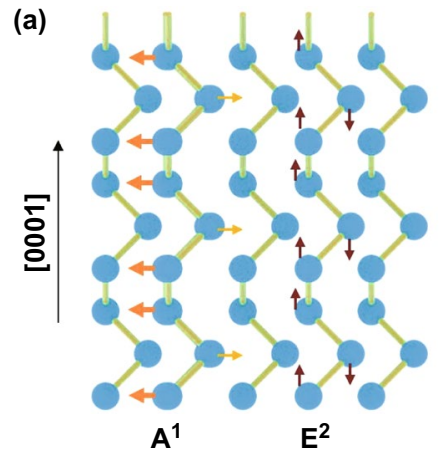

(1)

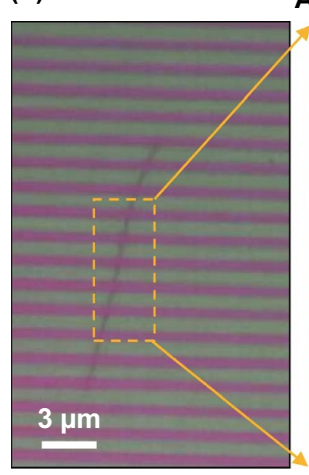

A1 Raman Intensity

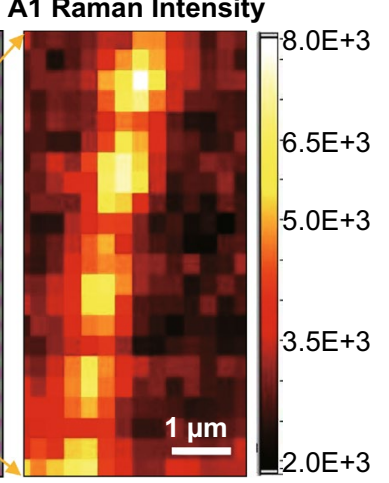

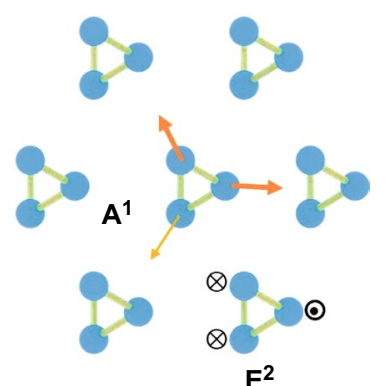

$\mathbf{E}^{2}$

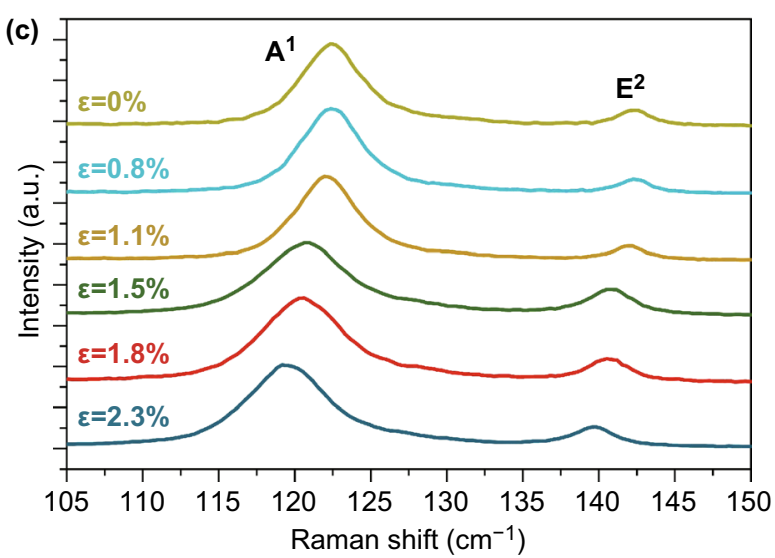

(d)
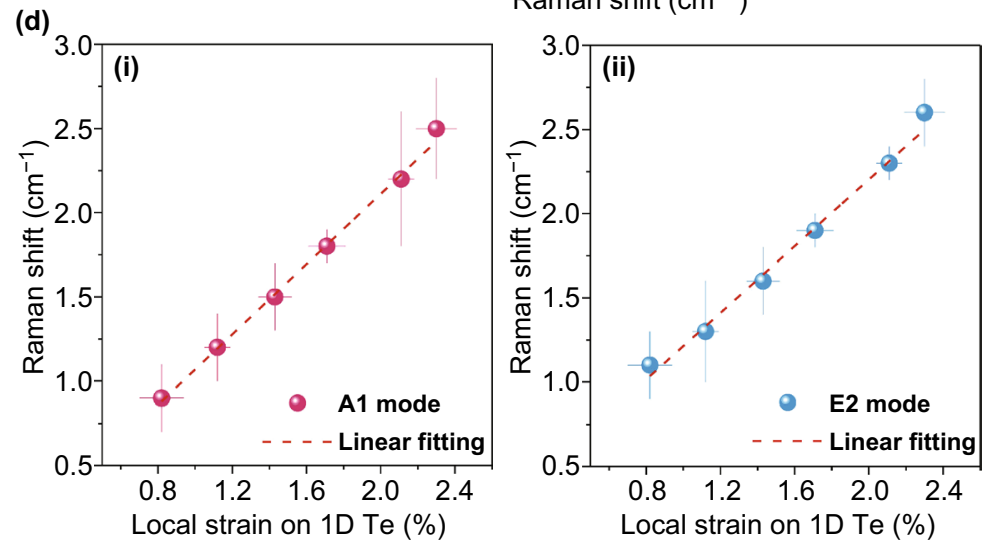

Fig. 4 Raman spectroscopy results of the strained 1D Te nanowire. a Schematic shows the main Raman-active modes in Te crystal. b Optical image and Raman intensity mapping of the deformed 1D Te nanowire. c Raman spectra of the deformed 1D with different local strain. d Quantitative analysis of the Raman frequency shift of the deformed 1D Te with different local strains. The average values from 3 technical replicates are indicated, and error bars represent one standard deviation for each set of replicates

peak strains (bright spots in the Raman mapping in Fig. 4b). Figure $4 \mathrm{c}$ shows the strain-engineered Raman shifts in the $A^{1}$ and $E^{2}$ modes when the local peak strain was increased from 0 to $2.3 \%$. When the Te nanowire (diameter $40 \mathrm{~nm}$ ) was naturally deposited on the substrate without introducing the nanoimprinting strain, two Raman-active modes locating at $121 \mathrm{~cm}^{-1}\left(A^{1}\right.$-mode) and $143 \mathrm{~cm}^{-1}\left(E^{2}\right.$-mode) were identified, which is consistent with previous literature and our results in Fig. 1. When we increased the local peak strains, both modes showed a significant red shift in the Raman peaks (Fig. 4c). The $A^{1}$-mode peak shifted to $117.6 \mathrm{~cm}^{-1}$, and the $E^{2}$-mode peak shifted to $140.3 \mathrm{~cm}^{-1}$ when the strain was increased to $2.3 \%$. We plotted the Raman frequency shift as the function of the local strains (Fig. 4d), and the results revealed a linear trend. The linear relationship between the Raman frequency shift and the uniaxial tensile strain $\varepsilon$ is given by $\Delta \omega=\gamma \varepsilon[105,106]$ where $\gamma$ is the phonon deformation potential coefficient, $\varepsilon$ is the uniaxial tensile strain on the materials, and $\Delta \omega$ is the phonon frequency shift. The rate of the shift is determined to be approximately $1.1 \mathrm{~cm}^{-1} \%^{-1}$ for $A^{1}$-mode [Fig. $\left.4 \mathrm{~d}(\mathrm{i})\right]$ and $1.2 \mathrm{~cm}^{-1} \%^{-1}$ for $E^{2}$-mode [Fig. 4d(ii)], respectively. These results are also consistent with our previous results for 2D Te [107]. The response of Raman spectra with different strains can be explained by examining the intrinsic vibration modes of Te [97] and the electro-optic interactions in Te [106]. When the tensile strain was applied along the [0001] direction of the Te nanowire, the length for the Te-Te covalent bond was elongated (Fig. 1e), which weakens the interatomic interaction [107, 108]. As a result, the stretching along the [0001] helical chain direction became softening, leading to a decrease in the vibration frequency of $E^{2}$-mode [109, 110]. Meanwhile, this softening effect leads to the weaker Te atom vibration in (10ī0) plane, resulting in a decreased vibration frequency of Te helical chain breathing in this plane, which is associated with the observed redshift in $A^{1}$-mode peak. 


\section{Conclusions}

In summary, we systematically investigated the strain-engineered optical properties in 1D chiral semiconductor tellurium, through applying a facile resist-free low-temperature nanoimprinting strategy to induce parallel nanoshaping in solution-grown ultralong 1D Te nanowires using commercial CD and DVD substrates. The observed Raman spectra from the chiral-chain lattice of 1D Te reveal the anisotropic lattice vibration under the corresponding straining conditions. We performed synergistic efforts combining theoretical and experimental investigations to elucidate the impacts of strain engineering on the lattice structure and optical properties of the $1 \mathrm{D}$ helical chain tellurium. The nanoimprinting-introduced elastic strain in Te nanowires is self-sustained and tunable, which could be further utilized for modulating the electrical, electrical-optical, mechanical, and other material properties of Te nanowires. The exquisite strain engineering on 1D Te could open up opportunities in flexible, wearable integrated electronic systems, as well as promising elastic strain engineering that takes advantage of the drastically changed electronic and optical properties under large lattice strain. The experimental platform can also enable the exquisite mechanical control in 1D Te and other nanomaterials with substrate-induced, on-demand, and controlled strains.

\section{Materials and Methods}

\subsection{Materials}

PVP, hydrazine hydrate $(85 \%, w / w)$, acetone, ethylene glycol (EG), aqueous ammonia solution (25-28\%, w/w), $N, N-$ dimethylformamide (DMF), $\mathrm{CHCl}_{3}$ were purchased from Sigma-Aldrich. $\mathrm{Na}_{2} \mathrm{TeO}_{3}(97 \%)$ was purchased from Alfa Aesar. All chemical reagents were used without further purification. Double-distilled deionized water (DIW; 18.2 megaohms) was used for the synthesis.

\subsection{Synthesis of Te Nanowires}

In the typical synthesis, $0.1 \mathrm{~g} \mathrm{Na}_{2} \mathrm{TeO}_{3}$ and $1 \mathrm{~g}$ PVP were put into $15 \mathrm{~mL}$ double-distilled water and $15 \mathrm{~mL}$ EG at room temperature under magnetic stirring to form a homogeneous solution. The resulting solution was poured into a
50-mL Teflon-lined steel autoclave, which was then filled with aqueous ammonia solution and hydrazine hydrate. The autoclave was sealed and maintained at $180^{\circ} \mathrm{C}$ for $3 \mathrm{~h}$. Then, the autoclave was cooled to room temperature naturally. The resulting blue products were precipitated by centrifuge at $5000 \mathrm{rpm}$ for 5 min and washed with distilled water and acetone (to remove any ions remaining in the final product).

\subsection{Langmuir-Blodgett (LB) Transfer of 1D Te}

The hydrophilic Te nanowires can be transferred to substrates by the Langmuir-Blodgett (LB) technique. The washed Te nanowires were mixed with a certain volume ratio of $\mathrm{N}, \mathrm{N}$-dimethylformamide (DMF), and $\mathrm{CHCl}_{3}$. Then, the above solution was dropped into the deionized water. Then, Te nanowire monolayer was prepared at $25^{\circ} \mathrm{C}$ using an LB trough (Nima Technology, 312D). The trough was filled with Millipore Milli-Q water (resistivity of $18.2 \mathrm{Mohm}$ $\mathrm{cm}$ ) until it brimmed just over the top by about $2 \mathrm{~mm}$. Five milliliters of the solution of Te nanowires were precipitated by adding $12 \mathrm{~mL}$ of acetone and centrifuging at $5000 \mathrm{rpm}$ for $5 \mathrm{~min}$. After $30 \mathrm{~min}$, the monolayer Te nanowire can be transferred to any substrates. The monolayer was lifted at a pressure of $23 \mathrm{mN} \mathrm{m}^{-1}$, and the deposition speed was maintained at $4 \mathrm{~mm} \mathrm{~min}^{-1}$. The centimeter-scale assembly of nanowires can be realized with controlled density, orientation, and spacing $[99,100,111]$. The floated nanowires can be parallelly aligned by a controlled barrier.

\subsection{Nanoimprinting Process}

Before nanoimprinting, the polyester terephthalate thin film was spin-coated on a silicon wafer with $4000 \mathrm{rpm}$ in $45 \mathrm{~s}$. The commercial blank compact disks were sonicated in acetone and water. The nanoimprinting was performed using Nanonex NX 2000.

\subsection{DFT Calculations}

Vienna Ab initio Simulation Package (VASP) 5.4.4 $[112,113]$ was used for performing DFT calculations with the projector augmented wave method [114]. The exchange-correlation functional of OptPBE-vdW [115] was used to take into account van der Waals interactions. The plane-wave basis set cutoff was $400 \mathrm{eV}$, and a 
Gamma-centered k-point mesh was set to $5 \times 5 \times 3$. Gaussian smearing of $0.1 \mathrm{eV}$ was used. The electronic wavefunction convergence threshold was $10^{-8} \mathrm{eV}$, and a $0.01 \mathrm{Ev} \AA^{-1}$ convergence criterion for forces on each atom was adopted.

\subsection{Characterization}

The thickness and morphology of the substrate surface were determined by AFM (Keysight 5500). High-resolution STEM/ TEM imaging and SAED have been performed using a probecorrected JEM-ARM 200F (JEOL USA, Inc.) operated at $200 \mathrm{kV}$, and EDS has been collected by an X-MaxN100TLE detector (Oxford Instruments). A field emission scanning electron microscope (Hitachi S-4800 Field Emission SEM) was used to characterize the morphologies of Te nanowires.

Acknowledgements W.Z.W. acknowledges the College of Engineering and School of Industrial Engineering at Purdue University for startup support. W.Z.W. was partially supported by the National Science Foundation under Grant CMMI-1762698. G.J.C. acknowledges financial assistance from ONR NEPTUNE program National Science Foundation under Grant CMMI-1538360. Q.W. and M.J.K. were supported by the Louis Beecherl, Jr. Endowment Funds. P.L. Acknowledges the College of Engineering and School of Materials Engineering at Purdue University for startup support. The computational research was supported through computational resources provided by the Information Technology department at Purdue University. The authors gratefully acknowledge the support and help from Professor Alexander Wei and his group in the LB assembly.

Open Access This article is licensed under a Creative Commons Attribution 4.0 International License, which permits use, sharing, adaptation, distribution and reproduction in any medium or format, as long as you give appropriate credit to the original author(s) and the source, provide a link to the Creative Commons licence, and indicate if changes were made. The images or other third party material in this article are included in the article's Creative Commons licence, unless indicated otherwise in a credit line to the material. If material is not included in the article's Creative Commons licence and your intended use is not permitted by statutory regulation or exceeds the permitted use, you will need to obtain permission directly from the copyright holder. To view a copy of this licence, visit http://creativecommons.org/licenses/by/4.0/.

\section{References}

1. B. Wu, A. Heidelberg, J.J. Boland, Mechanical properties of ultrahigh-strength gold nanowires. Nat. Mater. 4, 525-529 (2005). https://doi.org/10.1038/nmat1403
2. R.N. Barnett, U. Landman, Cluster-derived structures and conductance fluctuations in nanowires. Nature 387, 788-791 (1997). https://doi.org/10.1038/42904

3. E.W. Wong, P.E. Sheehan, C.M. Lieber, Nanobeam mechanics: elasticity, strength, and toughness of nanorods and nanotubes. Science 277, 1971-1975 (1997). https://doi. org/10.1126/science.277.5334.1971

4. C. Herring, J.K. Galt, Elastic and plastic properties of very small metal specimens. Phys. Rev. 85, 1060-1061 (1952). https://doi.org/10.1103/PhysRev.85.1060.2

5. J. Song, X. Wang, E. Riedo, Z.L. Wang, Elastic property of vertically aligned nanowires. Nano Lett. 5, 1954-1958 (2005). https://doi.org/10.1021/n1051334v

6. M.J. Gordon, T. Baron, F. Dhalluin, P. Gentile, P. Ferret, Size effects in mechanical deformation and fracture of cantilevered silicon nanowires. Nano Lett. 9, 525-529 (2009). https://doi.org/10.1021/n1802556d

7. R.A. Minamisawa, M.J. Süess, R. Spolenak, J. Faist, C. David, J. Gobrecht, K.K. Bourdelle, H. Sigg, Top-down fabricated silicon nanowires under tensile elastic strain up to $4.5 \%$. Nat. Commun. 3, 1096 (2012). https://doi. org/10.1038/ncomms 2102

8. M.W. Larsson, J.B. Wagner, M. Wallin, P. Håkansson, L.E. Fröberg, L. Samuelson, L.R. Wallenberg, Strain mapping in free-standing heterostructured wurtzite InAs/InP nanowires. Nanotechnology 18, 015504 (2006). https://doi. org/10.1088/0957-4484/18/1/015504

9. R. Adelung, O.C. Aktas, J. Franc, A. Biswas, R. Kunz et al., Strain-controlled growth of nanowires within thinfilm cracks. Nat. Mater. 3, 375-379 (2004). https://doi. org/10.1038/nmat1128

10. A. Nakamura, K. Matsunaga, J. Tohma, T. Yamamoto, Y. Ikuhara, Conducting nanowires in insulating ceramics. Nat. Mater. 2, 453-456 (2003). https://doi.org/10.1038/nmat920

11. G. Cheng, C. Miao, Q. Qin, J. Li, F. Xu et al., Large anelasticity and associated energy dissipation in single-crystalline nanowires. Nat. Nanotechnol. 10, 687-691 (2015). https:// doi.org/10.1038/nnano.2015.135

12. Q. Qin, S. Yin, G. Cheng, X. Li, T.-H. Chang et al., Recoverable plasticity in penta-twinned metallic nanowires governed by dislocation nucleation and retraction. Nat. Commun. 6, 5983 (2015). https://doi.org/10.1038/ncomms6983

13. G. Yu, A. Cao, C.M. Lieber, Large-area blown bubble films of aligned nanowires and carbon nanotubes. Nat. Nanotechnol. 2, 372-377 (2007). https://doi.org/10.1038/nnano .2007 .150

14. I.A. Goldthorpe, A.F. Marshall, P.C. McIntyre, Synthesis and strain relaxation of Ge-core/Si-shell nanowire arrays. Nano Lett. 8, 4081-4086 (2008). https://doi.org/10.1021/ n1802408y

15. G. Signorello, E. Lörtscher, P.A. Khomyakov, S. Karg, D.L. Dheeraj et al., Inducing a direct-to-pseudodirect bandgap transition in wurtzite GaAs nanowires with uniaxial stress. Nat. Commun. 5, 3655 (2014). https://doi.org/10.1038/ ncomms 4655 
16. J. Diao, K. Gall, M.L. Dunn, Surface-stress-induced phase transformation in metal nanowires. Nat. Mater. 2, 656-660 (2003). https://doi.org/10.1038/nmat977

17. J. Johansson, L.S. Karlsson, C. Patrik, T. Svensson, T. Mårtensson, B.A. Wacaser et al., Structural properties of $\langle 111\rangle$ B-oriented III-V nanowires. Nat. Mater. 5, 574-580 (2006). https://doi.org/10.1038/nmat1677

18. D.K. Ferry, Nanowires in nanoelectronics. Science 319, 579-580 (2008). https://doi.org/10.1126/science.1154446

19. K. Tomioka, M. Yoshimura, T. Fukui, A III-V nanowire channel on silicon for high-performance vertical transistors. Nature 488, 189-192 (2012). https://doi.org/10.1038/natur e11293

20. N.S. Malvankar, M. Vargas, K.P. Nevin, A.E. Franks, C. Leang et al., Tunable metallic-like conductivity in microbial nanowire networks. Nat. Nanotechnol. 6, 573-579 (2011). https://doi.org/10.1038/nnano.2011.119

21. C. Feng, S. Wang, L. Yin, X. Li, M. Yao et al., Significant strain-induced orbital reconstruction and strong interfacial magnetism in $\mathrm{TiNi}(\mathrm{Nb}) /$ ferromagnet/oxide heterostructures via oxygen manipulation. Adv. Funct. Mater. 28, 1803335 (2018). https://doi.org/10.1002/adfm.201803335

22. P.E. Kremer, A.C. Dada, P. Kumar, Y. Ma, S. Kumar, E. Clarke, B.D. Gerardot, Strain-tunable quantum dot embedded in a nanowire antenna. Phys. Rev. B 90, 201408 (2014). https://doi.org/10.1103/PhysRevB.90.201408

23. P. Krogstrup, N.L.B. Ziino, W. Chang, S.M. Albrecht, M.H. Madsen et al., Epitaxy of semiconductor-superconductor nanowires. Nat. Mater. 14, 400-406 (2015). https://doi. org/10.1038/nmat4176

24. A. Smogunov, A. Dal Corso, A. Delin, R. Weht, E. Tosatti, Colossal magnetic anisotropy of monatomic free and deposited platinum nanowires. Nat. Nanotechnol. 3, 22-25 (2008). https://doi.org/10.1038/nnano.2007.419

25. A. Bezryadin, C.N. Lau, M. Tinkham, Quantum suppression of superconductivity in ultrathin nanowires. Nature 404, 971-974 (2000). https://doi.org/10.1038/35010060

26. K. Takei, T. Takahashi, J.C. Ho, H. Ko, A.G. Gillies et al., Nanowire active-matrix circuitry for low-voltage macroscale artificial skin. Nat. Mater. 9, 821-826 (2010). https://doi. org/10.1038/nmat2835

27. S. Xu, B.J. Hansen, Z.L. Wang, Piezoelectric-nanowire-enabled power source for driving wireless microelectronics. Nat. Commun. 1, 93 (2010). https://doi.org/10.1038/ncomms1098

28. G. Zheng, F. Patolsky, Y. Cui, W.U. Wang, C.M. Lieber, Multiplexed electrical detection of cancer markers with nanowire sensor arrays. Nat. Biotechnol. 23, 1294-1301 (2005). https ://doi.org/10.1038/nbt1138

29. Z.L. Wang, J. Song, Piezoelectric nanogenerators based on zinc oxide nanowire arrays. Science 312, 242-246 (2006). https://doi.org/10.1126/science.1124005

30. W. Wu, Y. Wei, Z.L. Wang, Strain-gated piezotronic logic nanodevices. Adv. Mater. 22, 4711-4715 (2010). https://doi. org/10.1002/adma.201001925
31. W. Wu, X. Wen, Z.L. Wang, Taxel-addressable matrix of vertical-nanowire piezotronic transistors for active and adaptive tactile imaging. Science 340, 952-957 (2013). https://doi. org/10.1126/science.1234855

32. W. Wu, Z.L. Wang, Piezotronic nanowire-based resistive switches as programmable electromechanical memories. Nano Lett. 11, 2779-2785 (2011). https://doi.org/10.1021/ nl201074a

33. T. Ling, D.-Y. Yan, H. Wang, Y. Jiao, Z. Hu et al., Activating cobalt(II) oxide nanorods for efficient electrocatalysis by strain engineering. Nat. Commun. 8, 1509 (2017). https ://doi.org/10.1038/s41467-017-01872-y

34. M. Luo, S. Guo, Strain-controlled electrocatalysis on multimetallic nanomaterials. Nat. Rev. Mater. 2, 17059 (2017). https://doi.org/10.1038/natrevmats.2017.59

35. L. Bu, S. Guo, X. Zhang, X. Shen, D. Su et al., Surface engineering of hierarchical platinum-cobalt nanowires for efficient electrocatalysis. Nat. Commun. 7, 11850 (2016). https://doi.org/10.1038/ncomms11850

36. R. He, P. Yang, Giant piezoresistance effect in silicon nanowires. Nat. Nanotechnol. 1, 42-46 (2006). https://doi. org/10.1038/nnano.2006.53

37. W. Wu, C. Pan, Y. Zhang, X. Wen, Z.L. Wang, Piezotronics and piezo-phototronics-from single nanodevices to array of devices and then to integrated functional system. Nano Today 8, 619-642 (2013). https://doi.org/10.1016/j.nanto d.2013.11.002

38. Y. Yue, P. Liu, Z. Zhang, X. Han, E. Ma, Approaching the theoretical elastic strain limit in copper nanowires. Nano Lett. 11, 3151-3155 (2011). https://doi.org/10.1021/nl201233u

39. C. Marini, D. Chermisi, M. Lavagnini, D. Di Castro, C. Petrillo et al., High-pressure phases of crystalline tellurium: a combined Raman and ab initio study. Phys. Rev. B 86, 064103 (2012). https://doi.org/10.1103/PhysRevB.86.064103

40. D. Shiri, Y. Kong, A. Buin, M.P. Anantram, Strain induced change of bandgap and effective mass in silicon nanowires. Appl. Phys. Lett. 93, 073114 (2008). https://doi. org/10.1063/1.2973208

41. H.S. Park, Surface stress effects on the critical buckling strains of silicon nanowires. Comput. Mater. Sci. 51, 396-401 (2012). https://doi.org/10.1016/j.commatsci.2011.07.059

42. F. Salazar, A. Trejo-Baños, A. Miranda, L.A. Pérez, M. Cruz-Irisson, Interstitial sodium and lithium doping effects on the electronic and mechanical properties of silicon nanowires: a DFT study. J. Mol. Model. 25, 338 (2019). https://doi.org/10.1007/s00894-019-4239-5

43. M.N. Esfahani, Surface stress effects on the mechanical properties of silicon nanowires: a molecular dynamics simulation. J. Appl. Phys. 125, 135101 (2019). https://doi. org/10.1063/1.5089613

44. H. Zhang, K.-Y. Fung, Y. Zhuang, K. Cao, J. Song, A. Hu, Y. Lu, Fracture of a silicon nanowire at ultra-large elastic strain. Acta Mech. 230, 1441-1449 (2019). https://doi. org/10.1007/s00707-017-2015-0 
45. M. Wölz, M. Ramsteiner, V.M. Kaganer, O. Brandt, L. Geelhaar, H. Riechert, Strain engineering of nanowire multi-quantum well demonstrated by raman spectroscopy. Nano Lett. 13, 4053-4059 (2013). https://doi.org/10.1021/nl401306q

46. M.M.J. Treacy, T.W. Ebbesen, J.M. Gibson, Exceptionally high Young's modulus observed for individual carbon nanotubes. Nature 381, 678-680 (1996). https://doi. org/10.1038/381678a0

47. W. Michael Cai, L. Juyoung, K. Pilgyu, C. Jonghyun, K. Peter, Y. Keong, N. SungWoo, 2D Mater. 4, 022002 (1996)

48. H. Jiang, D.-Y. Khang, J. Song, Y. Sun, Y. Huang, J.A. Rogers, Finite deformation mechanics in buckled thin films on compliant supports. Proc. Natl. Acad. Sci. 104, 1560715612 (2007). https://doi.org/10.1073/pnas.0702927104

49. S. Deng, A.V. Sumant, V. Berry, Strain engineering in two-dimensional nanomaterials beyond graphene. Nano Today 22, 14-35 (2018). https://doi.org/10.1016/j.nanto d.2018.07.001

50. R. Rafael, C.-G. Andrés, C. Emmanuele, G. Francisco, Strain engineering in semiconducting two-dimensional crystals. J. Phys.: Condens. Matter 27, 313201 (2015). https ://doi.org/10.1088/0953-8984/27/31/313201

51. D.Y. Khang, J.A. Rogers, H.H. Lee, Mechanical buckling: mechanics, metrology, and stretchable electronics. Adv. Funct. Mater. 19, 1526-1536 (2009). https://doi. org/10.1002/adfm.200801065

52. W.H. Koo, S.M. Jeong, F. Araoka, K. Ishikawa, S. Nishimura, T. Toyooka, H. Takezoe, Light extraction from organic light-emitting diodes enhanced by spontaneously formed buckles. Nat. Photonics 4, 222 (2010). https://doi. org/10.1038/nphoton.2010.7

53. C. Feng, Y. Li, L. Wang, Y. Cao, M. Yao et al., Giant strain control of antiferromagnetic moment in metallic FeMn by tuning exchange spring structure. Adv. Funct. Mater. 30, 1909708 (2020). https://doi.org/10.1002/adfm.201909708

54. L. Wang, C. Feng, Y. Li, F. Meng, S. Wang et al., Switchable magnetic anisotropy of ferromagnets by dual-ionmanipulated orbital engineering. ACS Appl. Mater. Interfaces. 11, 32475-32480 (2019). https://doi.org/10.1021/ acsami.9b09342

55. T.M.G. Mohiuddin, A. Lombardo, R.R. Nair, A. Bonetti, G. Savini et al., Uniaxial strain in graphene by Raman spectroscopy: G peak splitting, Grüneisen parameters, and sample orientation. Phys. Rev. B 79, 205433 (2009). https ://doi.org/10.1103/PhysRevB.79.205433

56. S.Y. Ryu, J. Xiao, W.I. Park, K.S. Son, Y.Y. Huang, U. Paik, J.A. Rogers, Lateral buckling mechanics in silicon nanowires on elastomeric substrates. Nano Lett. 9, 3214-3219 (2009). https://doi.org/10.1021/n1901450q

57. P. Gerd, C.-G. Andres, B. Michele, S.J.V.D.Z. Herre, A.S. Gary, K. Agnieszka, H. Thomas, S. Christian, K. Tobias, 2D Mater. 2, 015006 (2015)

58. N. Bowden, S. Brittain, A.G. Evans, J.W. Hutchinson, G.M. Whitesides, Spontaneous formation of ordered structures in thin films of metals supported on an elastomeric polymer. Nature 393, 146 (1998). https://doi.org/10.1038/30193
59. H. Li, A.W. Contryman, X. Qian, S.M. Ardakani, Y. Gong et al., Correction: Corrigendum: Optoelectronic crystal of artificial atoms in strain-textured molybdenum disulphide. Nat. Commun. 6, 7381 (2015). https://doi.org/10.1038/ ncomms 9080

60. Y. Xia, G.M. Whitesides, Soft lithography. Angew. Chem. Int. Ed. 37, 550-575 (1998). https://doi.org/10.1002/(SICI)15213773(19980316)37:5\%3c550:AID-ANIE550\%3e3.0.CO;2-G

61. S. Jin, Y. Wang, M. Motlag, S. Gao, J. Xu, Q. Nian, W. Wu, G.J. Cheng, Large-area direct laser-shock imprinting of a 3D biomimic hierarchical metal surface for triboelectric nanogenerators. Adv. Mater. 30, 1705840 (2018). https://doi. org/10.1002/adma.201705840

62. S. Jin, Z. Zhou, E.S.A. Sakr, M. Motlag, X. Huang et al., Scalable nanoshaping of hierarchical metallic patterns with multiplex laser shock imprinting using soft optical disks. Small 15, 1900481 (2019). https://doi.org/10.1002/ smll.201900481

63. K. Hölz, E. Schaudy, J. Lietard, M.M. Somoza, Multi-level patterning nucleic acid photolithography. Nat. Commun. 10, 3805 (2019). https://doi.org/10.1038/s41467-019-11670-3

64. T. Ito, S. Okazaki, Pushing the limits of lithography. Nature 406, 1027-1031 (2000). https://doi.org/10.1038/35023233

65. M. Horák, K. Bukvišová, V. Švarc, J. Jaskowiec, V. Křápek, T. Šikola, Comparative study of plasmonic antennas fabricated by electron beam and focused ion beam lithography. Sci. Rep. 8, 9640 (2018). https://doi.org/10.1038/s4159 8-018-28037-1

66. S.Y. Chou, P.R. Krauss, P.J. Renstrom, Imprint lithography with 25-nanometer resolution. Science 272, 85-87 (1996). https://doi.org/10.1126/science.272.5258.85

67. L.J. Guo, Nanoimprint lithography: methods and material requirements. Adv. Mater. 19, 495-513 (2007). https://doi. org/10.1002/adma.200600882

68. L.J. Guo, Recent progress in nanoimprint technology and its applications. J. Phys. D-Appl. Phys. 37, R123-R141 (2004). https://doi.org/10.1088/0022-3727/37/11/R01

69. S.Y. Chou, C. Keimel, J. Gu, Ultrafast and direct imprint of nanostructures in silicon. Nature 417, 835-837 (2002). https ://doi.org/10.1038/nature00792

70. P.R. Krauss, S.Y. Chou, Nano-compact disks with 400 Gbit/ in $2400 \mathrm{Gbit} / \mathrm{in} 2$ storage density fabricated using nanoimprint lithography and read with proximal probe. Appl. Phys. Lett. 71, 3174-3176 (1997). https://doi.org/10.1063/1.120280

71. L.T. Varghese, L. Fan, Y. Xuan, C. Tansarawiput, S. Kim, M. Qi, Resistless nanoimprinting in metal for plasmonic nanostructures. Small 9, 3778-3783 (2013). https://doi. org/10.1002/smll.201300168

72. Z. Li, Y. Gu, L. Wang, H. Ge, W. Wu et al., Hybrid nanoimprint-soft lithography with sub-15 nm resolution. Nano Lett. 9, 2306-2310 (2009). https://doi.org/10.1021/n19004892

73. S.Y. Chou, P.R. Krauss, Imprint lithography with sub$10 \mathrm{~nm}$ feature size and high throughput. Microelectron. Eng. 35, 237-240 (1997). https://doi.org/10.1016/S0167 $-9317(96) 00097-4$ 
74. A. von Hippel, Structure and conductivity in the VIb group of the periodic system. J. Chem. Phys. 16, 372-380 (1948). https://doi.org/10.1063/1.1746893

75. G. Arlt, P. Quadflieg, Electronic displacement in tellurium by mechanical strain. Phys. Status Solidi (B) 32, 687-689 (1969). https://doi.org/10.1002/pssb.19690320220

76. Y. Wang, G. Qiu, R. Wang, S. Huang, Q. Wang et al., Fieldeffect transistors made from solution-grown two-dimensional tellurene. Nat. Electron. 1, 228-236 (2018). https://doi. org/10.1038/s41928-018-0058-4

77. Y. Wang, R. de Souza Borges Ferreira, R. Wang, G. Qiu, G. $\mathrm{Li}$ et al., Data-driven and probabilistic learning of the process-structure-property relationship in solution-grown tellurene for optimized nanomanufacturing of high-performance nanoelectronics. Nano Energy 57, 480-491 (2019). https:// doi.org/10.1016/j.nanoen.2018.12.065

78. S. Lin, W. Li, Z. Chen, J. Shen, B. Ge, Y. Pei, Tellurium as a high-performance elemental thermoelectric. Nat. Commun. 7, 10287 (2016). https://doi.org/10.1038/ncomms10287

79. T.I. Lee, S. Lee, E. Lee, S. Sohn, Y. Lee et al., High-power density piezoelectric energy harvesting using radially strained ultrathin trigonal tellurium nanowire assembly. Adv. Mater. 25, 2920-2925 (2013). https://doi.org/10.1002/adma.20130 0657

80. Y. Wang, R. Wang, S. Wan, Q. Wang, M.J. Kim, D. Ding, $\mathrm{W}$. Wu, Scalable nanomanufacturing and assembly of chiralchain piezoelectric tellurium nanowires for wearable selfpowered cardiovascular monitoring. Nano Futures 3, 011001 (2019). https://doi.org/10.1088/2399-1984/aaf76f

81. S. Gao, Y. Wang, R. Wang, W. Wu, Piezotronic effect in 1D van der Waals solid of elemental tellurium nanobelt for smart adaptive electronics. Semicond. Sci. Technol. 32, 104004 (2017). https://doi.org/10.1088/1361-6641/aa8605

82. W. Wu, G. Qiu, Y. Wang, R. Wang, P. Ye, Tellurene: its physical properties,scalable nanomanufacturing, and device applications. Chem. Soc. Rev. 47, 7203-7212 (2018)

83. J. Ibers, Tellurium in a twist. Nat. Chem. 1, 508 (2009). https ://doi.org/10.1038/nchem.350

84. M. Wu, Y. Wang, S. Gao, R. Wang, Solution-synthesized chiral piezoelectric selenium nanowires for wearable self-powered human-integrated monitoring. Nano Energy 56, 693-699 (2019). https://doi.org/10.1016/j.nanoen.2018.12.003

85. A. Ben-Moshe, A.O. Govorov, G. Markovich, Enantioselective synthesis of intrinsically chiral mercury sulfide nanocrystals. Angew. Chem. Int. Ed. 52, 1275-1279 (2013). https:// doi.org/10.1002/anie.201207489

86. L. Zheng, X. Qiu, Z. Zhang, D. Zhu, Y. Xu, Solvothermal synthesis, crystal structure and luminescence property of a new 1D organic amine templated europium sulfate with helical chains. Inorg. Chem. Commun. 14, 906-909 (2011). https ://doi.org/10.1016/j.inoche.2011.03.027

87. X. Liu, H. Gao, J.E. Ward, X. Liu, B. Yin et al., Power generation from ambient humidity using protein nanowires. Nature 578, 550-554 (2020). https://doi.org/10.1038/s4158 6-020-2010-9
88. Y. Sun, B. Sun, J. He, G. Yang, C. Wang, Millimeters long super flexible $\mathrm{Mn}_{5} \mathrm{Si}_{3} @ \mathrm{SiO}_{2}$ electrical nanocables applicable in harsh environments. Nat. Commun. 11, 647 (2020). https ://doi.org/10.1038/s41467-019-14244-5

89. A. Aziz, T. Zhang, Y.-H. Lin, F. Daneshvar, H.-J. Sue, M.E. Welland, 1D copper nanowires for flexible printable electronics and high ampacity wires. Nanoscale 9, 13104-13111 (2017). https://doi.org/10.1039/C7NR02478A

90. H. Sun, Y. Zhang, J. Zhang, X. Sun, H. Peng, Energy harvesting and storage in $1 \mathrm{D}$ devices. Nat. Rev. Mater. 2, 17023 (2017). https://doi.org/10.1038/natrevmats.2017.23

91. Z. Lou, G. Shen, Flexible photodetectors based on 1D inorganic nanostructures. Adv. Sci. 3, 1500287 (2016). https:// doi.org/10.1002/advs.201500287

92. W. Wu, Z.L. Wang, Piezotronics and piezo-phototronics for adaptive electronics and optoelectronics. Nat. Rev. Mater. 1, 16031 (2016). https://doi.org/10.1038/natrevmats.2016.31

93. P. Song, H. Qin, H.-L. Gao, H.-P. Cong, S.-H. Yu, Selfhealing and superstretchable conductors from hierarchical nanowire assemblies. Nat. Commun. 9, 2786 (2018). https:// doi.org/10.1038/s41467-018-05238-w

94. H.-S. Qian, S.-H. Yu, J.-Y. Gong, L.-B. Luo, L.-F. Fei, High-quality luminescent tellurium nanowires of several nanometers in diameter and high aspect ratio synthesized by a poly(vinyl pyrrolidone)-assisted hydrothermal process. Langmuir 22, 3830-3835 (2006). https://doi.org/10.1021/ la0530211

95. J.-W. Liu, J. Xu, W. Hu, J.-L. Yang, S.-H. Yu, Systematic synthesis of tellurium nanostructures and their optical properties: from nanoparticles to nanorods, nanowires, and nanotubes. ChemNanoMat 2, 167-170 (2016). https://doi.org/10.1002/ cnma.201500206

96. K. Min-Seok, M. Xing-Hua, C. Ki-Hyun, J. Seung-Yeol, H. Kahyun, S. Yun-Mo, A generalized crystallographic description of all tellurium nanostructures. Adv. Mater. 30, 1702701 (2018). https://doi.org/10.1002/adma.201702701

97. A.S. Pine, G. Dresselhaus, Raman spectra and lattice dynamics of tellurium. Phys. Rev. B 4, 356-371 (1971). https://doi. org/10.1103/PhysRevB.4.356

98. A. Tao, F. Kim, C. Hess, J. Goldberger, R. He, Y. Sun, Y. Xia, P. Yang, Langmuir-Blodgett silver nanowire monolayers for molecular sensing using surface-enhanced raman spectroscopy. Nano Lett. 3, 1229-1233 (2003). https://doi. org/10.1021/n10344209

99. A.R. Tao, J. Huang, P. Yang, Langmuir-Blodgettry of nanocrystals and nanowires. Acc. Chem. Res. 41, 1662-1673 (2008). https://doi.org/10.1021/ar8000525

100. J.-W. Liu, J.-L. Wang, Z.-H. Wang, W.-R. Huang, S.-H. Yu, Manipulating nanowire assembly for flexible transparent electrodes. Angew. Chem. Int. Ed. 53, 13477-13482 (2014). https://doi.org/10.1002/anie.201408298

101. K. Ariga, Y. Yamauchi, T. Mori, J.P. Hill, 25th anniversary article: what can be done with the langmuir-blodgett method? Recent developments and its critical role in materials science. Adv. Mater. 25, 6477-6512 (2013). https://doi.org/10.1002/ adma.201302283 
102. C. Tian, H.-P. Ji, C.-Y. Zong, C.-H. Lu, Controlled fabrication of hierarchically microstructured surfaces via surface wrinkling combined with template replication. Chin. Chem. Lett. 26, 15-20 (2015). https://doi.org/10.1016/j.cclet.2014.10.003

103. A.J. Baca, J.-H. Ahn, Y. Sun, M.A. Meitl, E. Menard et al., Semiconductor wires and ribbons for high-performance flexible electronics. Angew. Chem. Int. Ed. 47, 5524-5542 (2008). https://doi.org/10.1002/anie.200703238

104. F. Xu, W. Lu, Y. Zhu, Controlled 3D buckling of silicon nanowires for stretchable electronics. ACS Nano 5, 672-678 (2011). https://doi.org/10.1021/nn103189z

105. X.-W. Fu, Z.-M. Liao, R. Liu, J. Xu, D. Yu, Size-dependent correlations between strain and phonon frequency in individual $\mathrm{ZnO}$ nanowires. ACS Nano 7, 8891-8898 (2013). https:// doi.org/10.1021/nn403378g

106. L. Kleinman, Deformation potentials in silicon. I. Uniaxial strain. Phys. Rev. 128, 2614-2621 (1962). https://doi. org/10.1103/PhysRev.128.2614

107. Y. Du, G. Qiu, Y. Wang, M. Si, X. Xu, W. Wu, P.D. Ye, One-dimensional van der Waals material tellurium: Raman spectroscopy under strain and magneto-transport. Nano Lett. 17, 3965-3973 (2017). https://doi.org/10.1021/acs.nanol ett.7b01717

108. W. Harrison, Elementary electronic structure (revised edition). (World Scientific Publishing Company, 2004)

109. Y. Du, J. Maassen, W. Wu, Z. Luo, X. Xu, P.D. Ye, Auxetic black phosphorus: a $2 \mathrm{D}$ material with negative
Poisson's ratio. Nano Lett. 16, 6701-6708 (2016). https:// doi.org/10.1021/acs.nanolett.6b03607

110. J.-W. Jiang, T. Chang, X. Guo, H.S. Park, Intrinsic negative Poisson's ratio for single-layer graphene. Nano Lett. 16, 5286-5290 (2016). https://doi.org/10.1021/acs.nanol ett.6b02538

111. D. Whang, S. Jin, Y. Wu, C.M. Lieber, Large-scale hierarchical organization of nanowire arrays for integrated nanosystems. Nano Lett. 3, 1255-1259 (2003). https://doi. org/10.1021/n10345062

112. G. Kresse, J. Furthmüller, Large-scale hierarchical organization of nanowire arrays for integrated nanosystems. Phys. Rev. B 54, 11169-11186 (1996). https://doi.org/10.1103/ PhysRevB.54.11169

113. G. Kresse, J. Furthmüller, Efficient iterative schemes for ab initio total-energy calculations using a plane-wave basis set. Comput. Mater. Sci. 6, 15-50 (1996). https://doi. org/10.1016/0927-0256(96)00008-0

114. G. Kresse, D. Joubert, From ultrasoft pseudopotentials to the projector augmented-wave method. Phys. Rev. B 59, 17581775 (1999). https://doi.org/10.1103/PhysRevB.59.1758

115. J. Klimeš, D.R. Bowler, A. Michaelides, Chemical accuracy for the van der Waals density functional. J. Phys. Condens. Matter 22, 022201 (2009). https://doi.org/10.1088/0953$8984 / 22 / 2 / 022201$ 